\title{
Two-Photon Excitation Photodynamic Therapy: Working Toward a New Treatment for Wet Age-Related Macular Degeneration
}

\author{
Ira Probodh and David Thomas Cramb \\ Department of Chemistry, University of Calgary \\ Canada
}

\section{Introduction}

Photodynamic therapy (PDT) exploits the cytotoxic effects of light-activated compounds to achieve spatially selective tissue eradication. It is used in treating a wide range of tumors (Lou et al., 2003), localized infections (Hamblin \& Hasan, 2004), and diseases like the wet form of age-related macular degeneration (Bressler \& Bressler, 2000). The treatment involves application of a non-toxic photosensitizer that is preferentially taken up by the target cells/tissue. Optical excitation of the photosensitizer produces reactive oxygen species that cause localised, apoptotic cell death. Herein we review the application of a new modality two-photon excitation-PDT (TPE-PDT) - to the treatment of wet age-related macular degeneration (wet-AMD). We show that the application of TPE-PDT, in conjunction with newly developed photosensitizers, has the potential to greatly improve therapy of wetAMD.

\subsection{Why two-photon photodynamic therapy (TPE-PDT)?}

\subsubsection{Photodynamic therapy (PDT)}

Wet-AMD is characterised by generation of blood vessels in the normally avascular retinal macula. The newly formed blood vessels leak fluid and/or blood under the macula, leading to rapid vision loss through damage to the photoreceptors (Rattner \& Nathans, 2006). PDT, using single photon activation of the photosensitizer Verteporfin (trade name Visudyne), has been used for the treatment of wet-AMD since 2000 (Bressler \& Bressler, 2000). In the clinic, verteporfin is first administered to patients through systemic injections (Soubrane \& Bressler, 2001), and the photosensitizer accumulates in areas of high cellular reproduction like the neovasculature in the retinal tissue. Photo-irradiation of the photosensitizer leads to a localised, Type II photoreaction associated with singlet oxygen generation (SchmidtErfurth \& Hasan, 2000). The photosensitizer absorbs a photon and is promoted to the excited singlet state that converts to an excited triplet state through intersystem crossing. An energy transfer between the triplet excited state of photosensitizer and naturally occurring triplet oxygen then produces reactive singlet oxygen. While Type I reactions involving radicals are also possible, PDT is generally accepted as occurring predominantly through the singlet oxygen mechanism. 
The PDT-induced vessel occlusion, in vivo, is generally attributed to singlet oxygen mediated direct vascular damage of blood vessel endothelium. This initiates a cascade of responses which include platelet aggregation, leukocyte adhesion, vascular permeabilization and vasoconstriction (Krammer, 2001). These, in turn, are expected to cause vascular occlusion.

The short lifetime of singlet oxygen (3.5 $\mu$ s in aqueous environment (Pervaiz, 2001)) ensures that the area affected by it is spatially confined to a small volume. It is estimated that singlet oxygen can diffuse to a distance of around 100nm or less (Skovsen et al., 2005) in vivo. PDT, thus, offers a relatively selective and non-invasive method to occlude the abnormal vascularization characteristic of wet-AMD. The stages in PDT for treatment of wet-AMD are diagrammed in Figure 1.
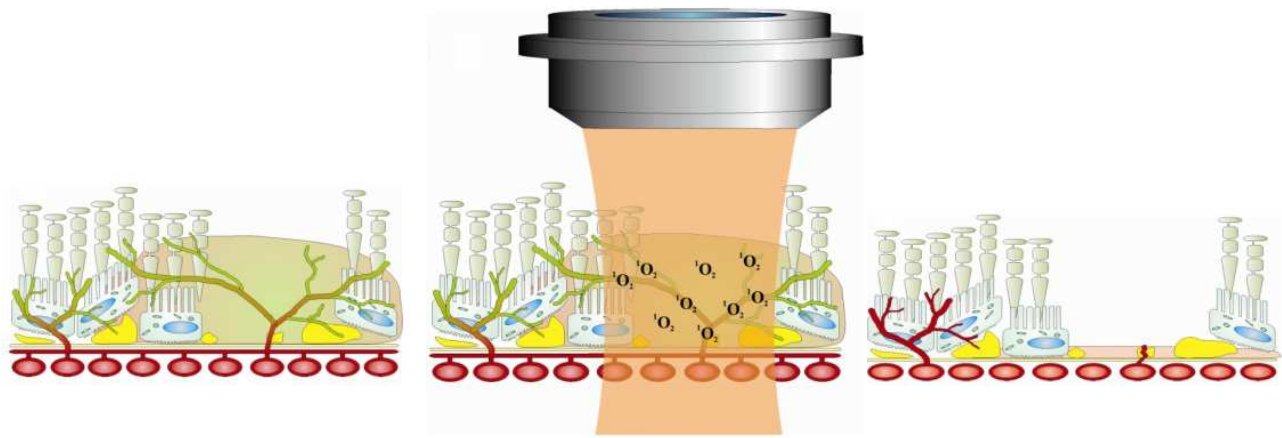

$$
\text { Verteporfin }+\mathrm{O}_{2}+h v
$$

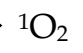

Vessel closure

Fig. 1. Visual representation of Verteporfin photodynamic therapy: injected verteporfin accumulates in the retinal neovasculature, where it is activated by illumination with a $680 \mathrm{~nm}$ laser beam. Laser activation leads to singlet oxygen production that in turn leads to vessel occlusion. [Adapted from PhD thesis of K. S. Samkoe (Samkoe, 2007)]

Currently, clinical PDT treatment involves excitation of Verteporfin with 689nm laser light that excites via a one-photon absorption peak in the so-called Q-band of the photosensitizer. The disadvantage of this treatment regime is that one-photon excitation can damage the over- and underlying tissues adjacent to the treated area, through excitation of photosensitizer present there (Reinke et al., 1999). This deleterious side-effect can be reduced by using two-photon excitation of photosensitizer.

\subsubsection{Two-photon excitation PDT (TPE-PDT)}

Two-photon excitation (TPE) of fluorophores is extensively utilized in confocal microscopy (Oheim et al., 2006; So et al., 2000). Because two-photon absorption cross-sections are very small, excitation requires high fluxes of light that can be achieved by using a tightly focused femtosecond laser beam as the light source. In TPE, a molecule is excited by simultaneous absorption of two photons of half the energy, or twice the wavelength, of one photon excitation. The first photon excites the molecule from its ground state to a virtual intermediate excited state. A second photon, simultaneously absorbed, promotes the 
molecule from the virtual intermediate state to the singlet excited state. The probability of this event is very small, and it is proportional to the square of the light intensity. Two-photon absorption, therefore, occurs only at the focal plane of a tightly focused laser beam (Goyan et al., 2001; Oheim et al., 2006). It should be noted that the excited states achieved by one and two-photon absorption are identical. The photophysical and photochemical properties of the photosensitizer are thus, unaffected by the mode of excitation (Goyan \& Cramb, 2000; Samkoe et al., 2006).

\section{One-photon excitation}
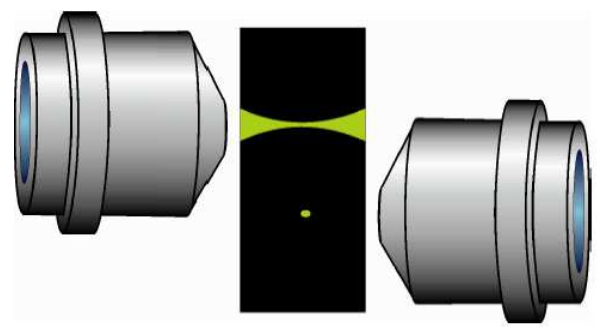

Two-photon excitation
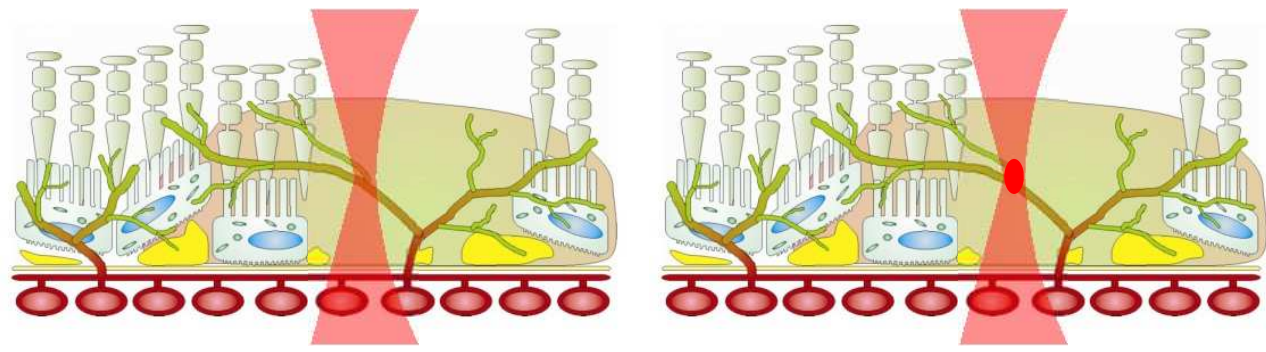

Fig. 2. In one photon excitation, photosensitizer activation occurs throughout the path of the laser beam, but in two-photon excitation, activation occurs only at the focus of the laser beam (red oval in right panel). The localised excitation in TPE-PDT is likely to cause less collateral damage. [Adapted from PhD thesis of K. S. Samkoe (Samkoe, 2007)]

Typically, excitation volumes of a few femtoliters can be achieved with two-photon absorption. This extremely confined excitation volume allows for high spatial selectivity. Use of a TPE treatment modality therefore, has the potential to selectively excite photosensitizer in the neovasculature, leaving the surrounding tissue unaffected (see Figure 2). In the following sections we review the experiments that demonstrate TPE-PDT in vitro and vessel occlusion in vivo with clinically approved and novel photosensitizers, and discuss the implications of its therapeutic use.

\section{TPE-PDT in vitro - Testing photosensitizers in cell-lines}

While in vivo experiments in animal models are essential to test and demonstrate the efficacy of drugs, it is very useful to quickly pre-screen drugs in cellular models before expending 
time and effort on laborious animal experiments. Cells can grow faster than animals, are inexpensive to maintain and easier to handle. In the context of PDT, they are particularly useful since cell death post-PDT treatment can be easily quantified.

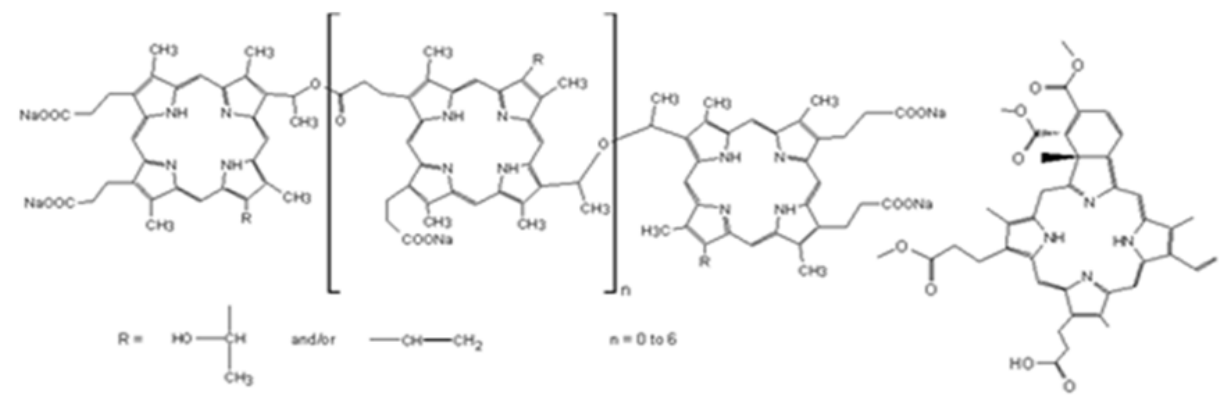

\section{Photofiin}

\section{Verteporfin}

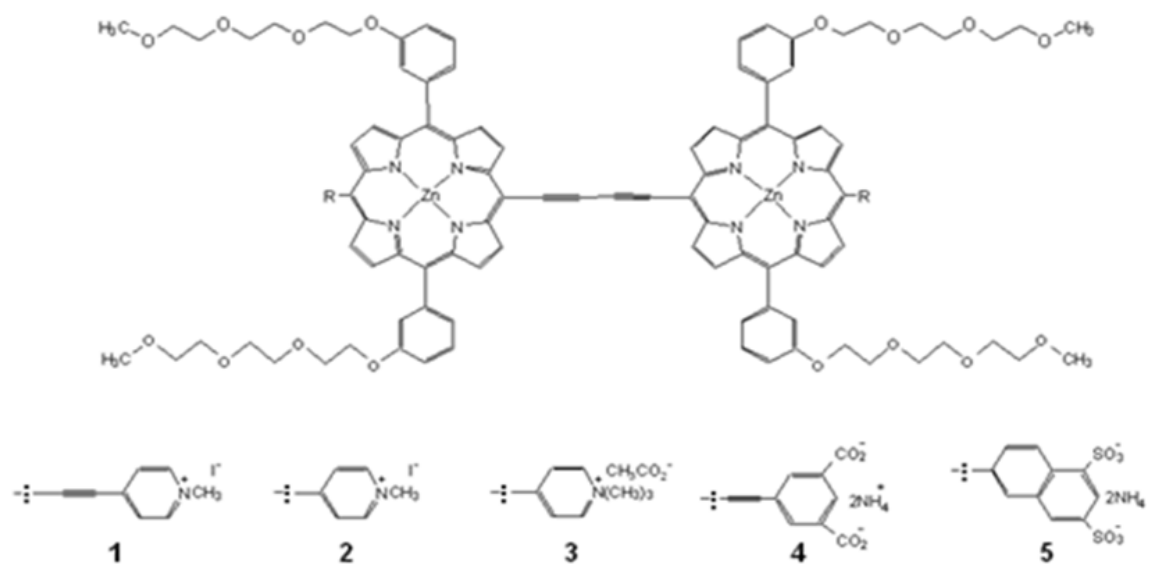

Porphyrin dimers

Fig. 3. The photosensitizers [Porphyrin dimers adapted from (Collins et al., 2008)]

Khurana et al (Khurana et al., 2007) have used endothelial cells to assess the TPE-PDT efficacy of photosensitizers approved for one photon PDT - namely, photofrin and verteporfin. They incubated a confluent monolayer of endothelial cells (YPEN-1) with each photosensitizer and performed TPE-PDT by irradiating the cell layer with the 865nm laser line of a femtosecond Ti:Sapphire laser at various output powers. TPE-PDT effect was quantified by using cell permeability stains to assess cell death post-treatment. The authors achieved TPE-PDT induced cell death with both photosensitizers, but Verteporfin was around seven times more effective, consistent with its higher two-photon cross-section. Using verteporfin and by varying the laser dose, they were also able to demonstrate the 
non-linear dependence of TPE-PDT on light intensity, providing unambiguous support of the involvement of two-photon processes in PDT.

While utilizing clinically approved photosensitizers for TPE-PDT is very attractive, their small two-photon absorption may be limiting in the clinical context. Collins et al (Collins et al., 2008) reported the use of "designer" TPE-PDT drugs - novel porphyrin dimers specifically designed to have high two-photon absorption cross section. The authors tested a series of porphyrin dimers for their PDT-induced cytotoxicity in a cancer cell line and demonstrated the higher TPE-PDT efficiency of dimer 1 (fig. 3) relative to verteporfin. Dimer 1 was then selected for in vivo TPE-PDT testing [section 3.2.2] and is a strong candidate for future development as a TPE-PDT photosensitizer.

\section{TPE-PDT in vivo}

\subsection{Complete occlusion of neovasculature in the chicken CAM}

\subsubsection{The CAM as a model for wet-AMD}

The chicken chorioallantoic membrane (CAM) is a transparent extra-embryonic membrane that grows against the inner wall of the developing egg. It is an external lung and waste exchange system for the embryo, and has a wide range of blood vessel sizes - from a few microns to several hundred microns in diameter (Patten, 1971). This makes it easy to find blood vessels that are similar in size to the neovasculature produced in the human eye during AMD (see Figure 4), and like the latter, the blood vessels are undergoing rapid angiogenesis between days 5 to 9 of gestation (Schlatter et al., 1997). The chicken embryo has a short gestation period and is easy to manipulate. It is as such, an inexpensive and useful model for the neovasculature occurring in wet-AMD.
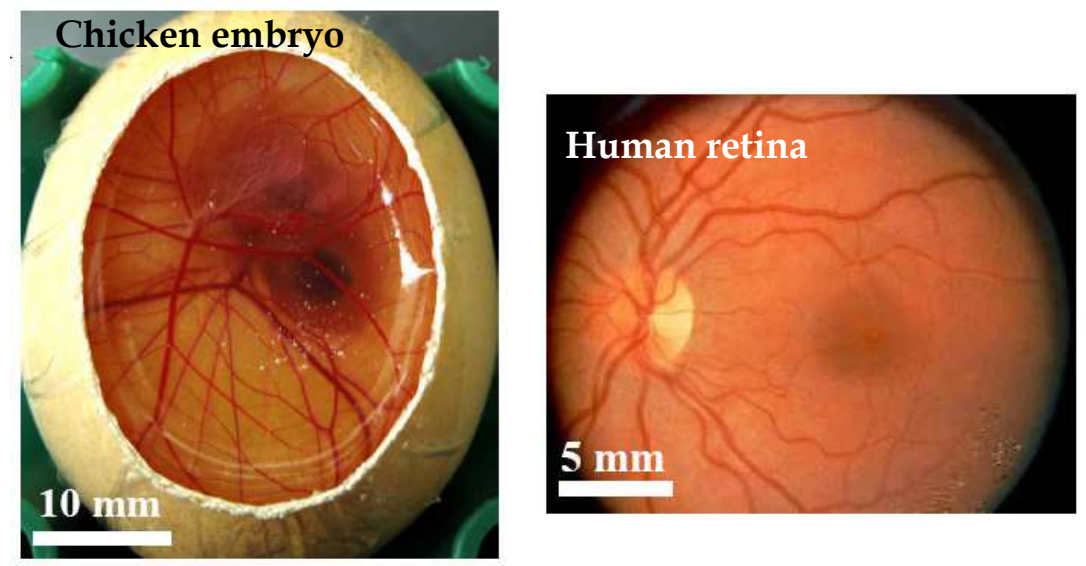

Fig. 4. The chicken chorio-allantoic membrane (CAM) exhibits blood vessels similar in size to those in the human eye, making it a good model for the neovasculature in wet-AMD. [Picture of chicken embryo reproduced with permission from Clancy et al., 2010, Chemical Physics Letters, 488, 99-111; picture of human retina reproduced from PhD thesis of K. S. Samkoe (Samkoe, 2007).] 


\subsubsection{Experimental set-up for PDT in the chicken embryo}

The following protocol to prepare the embryo was developed in the Cramb group (Samkoe et al., 2007). Chicken eggs are incubated at $37^{\circ} \mathrm{C}$ and $60 \%$ humidity for 9 days prior to the experiment. On day 4.5, the eggs are "windowed" by first draining 3-4ml of albumen from the blunt end using a syringe, and then cutting a small window in the egg-shell. The window is then covered by cellulose tape for the duration of the incubation, and widened before the experiment to facilitate injection.

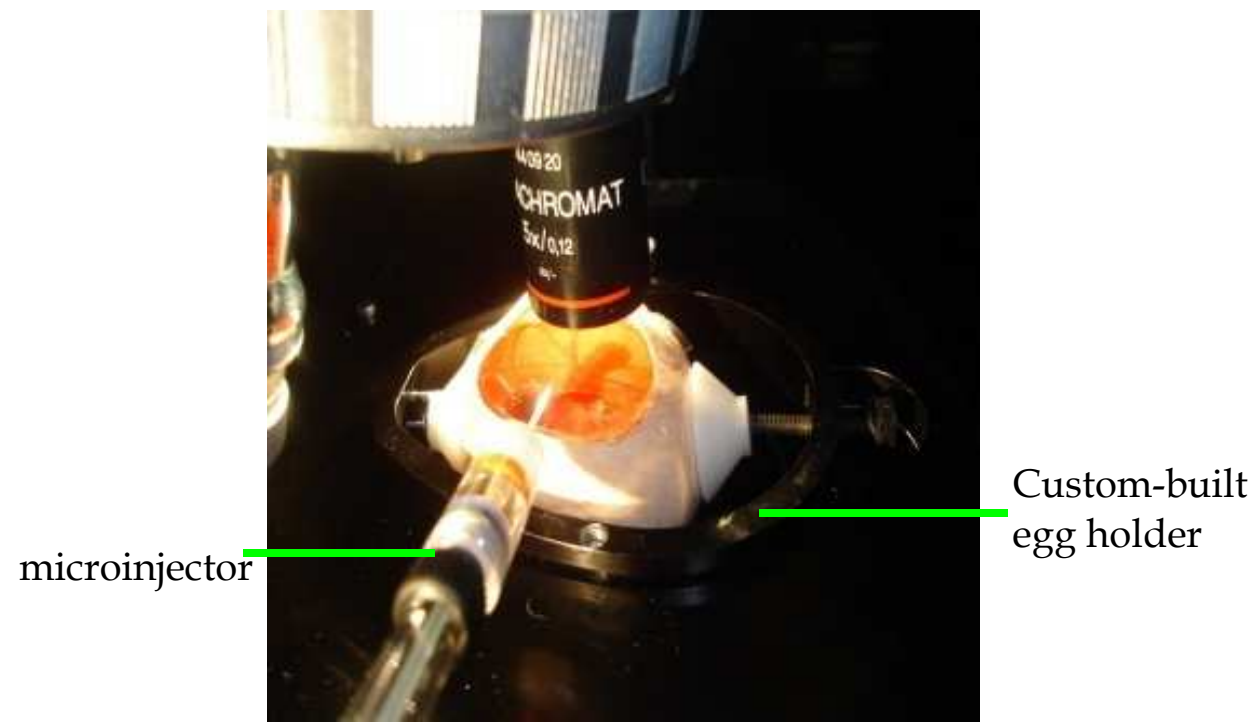

Fig. 5. TPE-PDT in the chicken CAM. Photosensitizer is injected with the microinjector attached to the custom-built stage and TPE-PDT is carried out by focusing the laser beam on the treated artery through the microscope objective. [Picture reproduced from PhD thesis of Y. Gregoriou (Gregoriou, 2011)]

For the TPE-PDT experiments, the embryo was mounted on an upright fluorescence microscope with a custom designed sample stage (Figure 5). Verteporfin was administered as a vesicle preparation, injected intrarterially or intravenously, using a microinjector attached to the sample stage. 10min after injection, PDT was performed by directing the light $(780 \mathrm{~nm})$ of a Ti:Sapphire laser (pulse duration $\sim 100 \mathrm{fsec}$ ) into the selected artery. Laser power of the incident light, duration of the laser illumination and the number of laser treatments were varied to achieve optimal PDT treatment. Vessel occlusion was monitored by taking video images of the treated areas before and after PDT treatment. For tracking long term occlusion, embryos were monitored for up to $6 \mathrm{hrs}$ after TPE-PDT treatment. For the multiple short laser treatments, each treatment was performed by focusing the laser beam on to the upper wall of the blood vessel for the required time, then moving it to another spot, close by, on the same artery.

\subsubsection{Vessel occlusion by TPE-PDT in the chicken embryo}

The first experiments demonstrating TPE-PDT-induced complete occlusion of blood vessels in the CAM occluded up to $15 \mu \mathrm{m}$ diameter arteries (Samkoe et al., 2007). A laser power of 
$38 \mathrm{~mW}$ (corresponding to a fluence of $1.1 \times 10^{8} \mathrm{~J} / \mathrm{cm}^{2}$ ) and treatment time of $5 \mathrm{~min}$ achieved complete occlusion of these small arteries immediately after the treatment. As expected, increasing the drug dose, the laser power, and/or the laser treatment time increases the efficacy of occlusion, presumably through increased singlet oxygen generation. By increasing the laser power and treatment times, it was also possible to achieve long-term occlusion of blood vessels. A minimum of $45 \mathrm{~mW}$ laser power and a laser treatment time of $3 \mathrm{~min}$ were required for long term occlusion of up to $50 \mu \mathrm{m}$ arteries (Khurana et al., 2009) (fig.6).

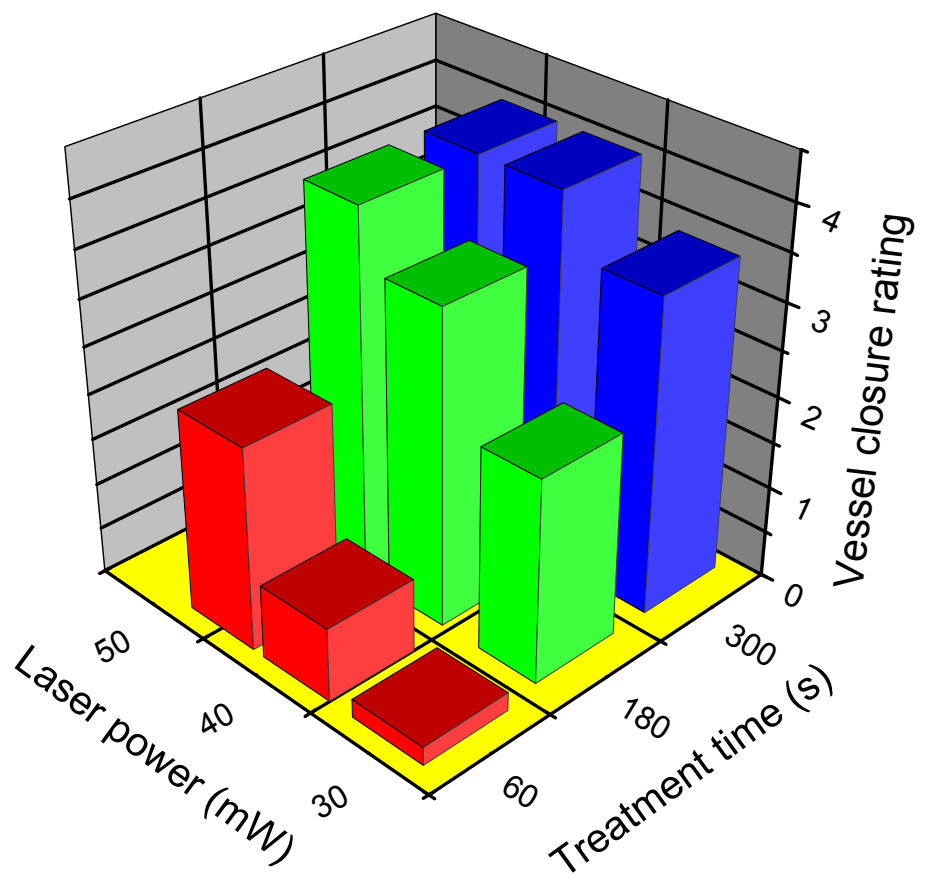

Fig. 6. Optimising laser dosage to occlude $50 \mu \mathrm{m}$ arteries. A vessel closure rating of 4 equals complete occlusion. [Adapted with permission from Khurana et al., (2009) Journal of Biomedical Optics, 14, 064006.]

Clearly, increasing the laser power and laser treatment times has the potential to improve occlusion efficacy of TPE-PDT and shut down larger feeder vessels. So is there a glass ceiling? The two major limiting factors are the laser's maximum power output which limits the maximum fluence, and the photosensitizer/oxygen concentration in the excitation volume, which limits the singlet oxygen generated during the treatment. In our experiments, it appears to be the latter, with the TPE-PDT efficacy levelling off at laser powers of $120 \mathrm{~mW}$ for the highest drug dose tried ( $2 \mathrm{mg}$ per $\mathrm{kg}$ of body weight). The option of increasing the treatment times is not preferred as longer treatment times reduce the long- 
term viability of the embryo and limit the usefulness of the model in terms of tracking longterm occlusion. Moreover, for patient compliance during TPE-PDT, shorter treatment times are preferred.

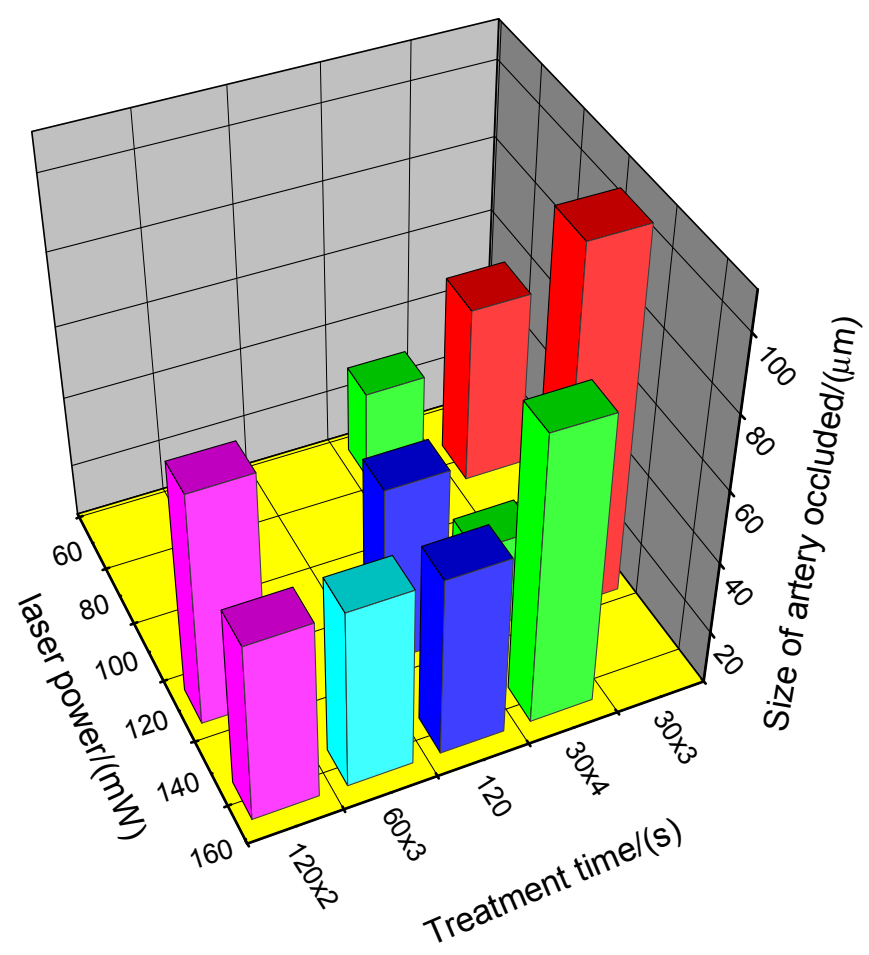

Fig. 7. Varying the laser dosage and treatment regimes to occlude large blood vessels shows that multiple short treatments are more useful than long single treatments in vessel occlusion.

Multiple short laser treatments, interspersed with 'dark' periods, could overcome these limitations by exciting a large number of photosensitizers in a short period, and then allowing the blood flow to replenish the ground state photosensitizer and/or oxygen during the 'dark' period. The examples in Fig. 7 and 8 clearly indicate that multiple short laser treatments are more efficacious than single long treatments to achieve long-term occlusion of large vessels. We were able to completely occlude up to $100 \mu \mathrm{m}$ sized arteries using laser power as low as $70 \mathrm{~mW}$ with $30 \mathrm{~s} \times 3$ laser treatments, whereas a laser power of $120 \mathrm{~mW}$ and 120s treatment time only succeeded in occluding up to $60 \mu \mathrm{m}$ arteries. Our data suggest that in the context of clinical verteporfin TPE-PDT treatment, it would be useful to investigate multiple short laser treatments to achieve optimal PDT efficiency and shut down large feeder vessels. 

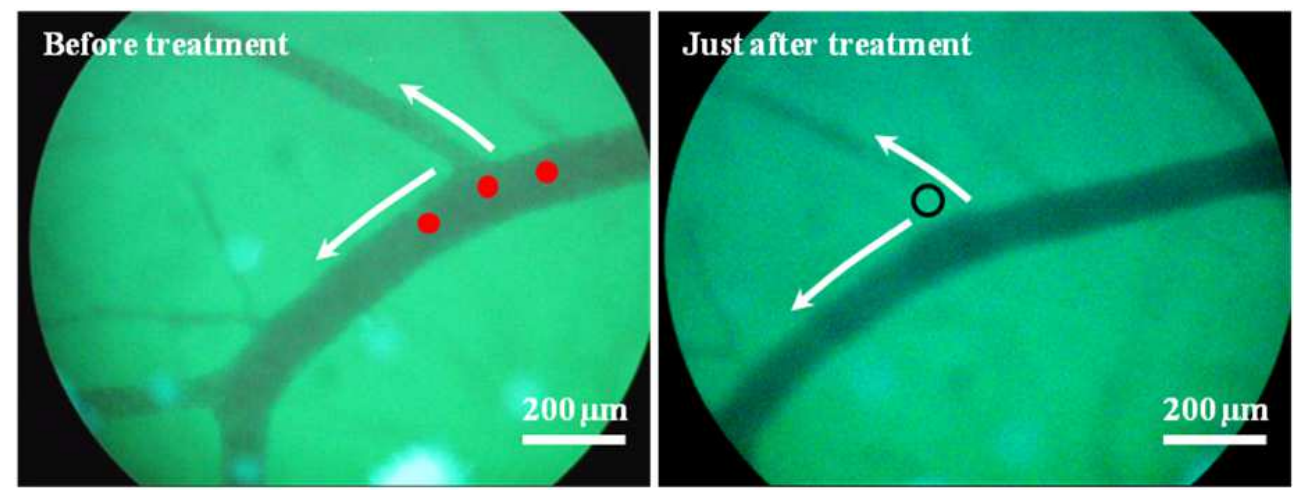

Fig. 8. Blood vessel occlusion after TPE-PDT. $2 \mathrm{mg} / \mathrm{kg}$ Verteporfin liposomal solution injected intravenously; $3 \times 30$ s treatments with $70 \mathrm{~mW}$ laser achieved almost complete occlusion of a $55 \mu \mathrm{m}$ artery immediately after treatment. (The red dots mark the approximate positions of the laser beam during TPE-PDT treatment)

\subsection{Vascular occlusion in the mouse window model}

\subsubsection{The mouse window chamber model for testing vascular occlusion}

Khurana et al (Khurana et al., 2009) have investigated TPE-PDT in a murine chamber window model. Surgical placement of a transparent window $(1 \mathrm{~cm}$ diameter) into the dorsal skin of a mouse allows for direct visualization of skin vasculature and administration of PDT treatment under a confocal laser scanning microscope (see Figure 9). This has the advantage of using a more robust animal model that allows for tracking of vascular occlusion on the long term - up to $25 \mathrm{hrs}$. With the chicken CAM model, it is typically possible to track vessel occlusion only up to 6-7hrs after the treatment. The disadvantage of using the mouse model for wet-AMD is that the blood vessels tested are normal healthy vessels, not leaky neovasculature of the type found in wet-AMD and the chicken CAM.
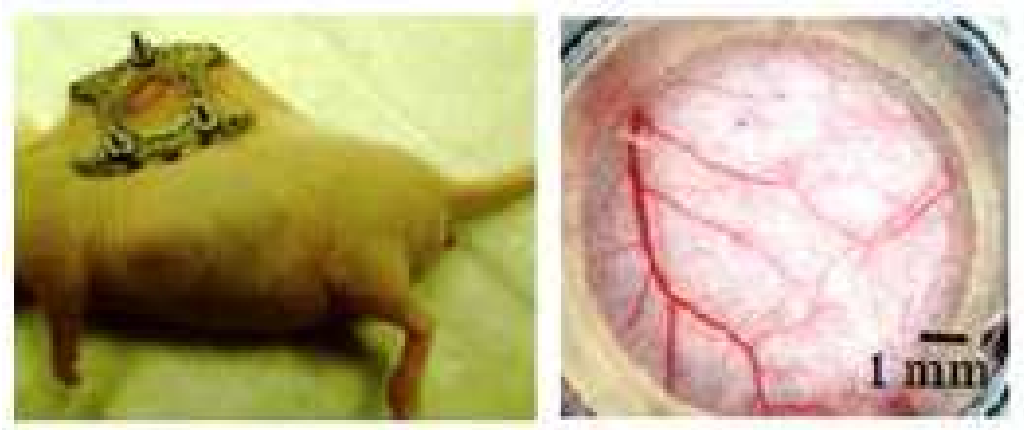

Fig. 9. The murine window chamber model. Nude mouse with surgically implanted window (left), vasculature visible through the window (right) [Reproduced with permission from Khurana et al., (2009) Journal of Biomedical Optics, 14, 064006] 


\subsubsection{Vessel occlusion by TPE-PDT in the windowed mouse}

Khurana et al (Khurana et al., 2009) tested two different photosensitizers for TPE-PDT verteporfin and a novel porphyrin dimer specifically designed for larger two-photon absorption. For TPE-PDT, a small area $\left(80 \times 80 \mu \mathrm{m}^{2}\right)$ on the selected blood vessel was raster scanned with the appropriate laser $(865 \mathrm{~nm}$ for verteporfin and $920 \mathrm{~nm}$ for the porphyrin dimer). A range of photosensitizer and light doses were investigated in order to obtain the optimal value of drug-light product (product of drug concentration and light fluence) and compare TPE-PDT to conventional one photon excitation PDT. The authors focused on complete occlusion of $40-50 \mu \mathrm{m}$ diameter arteries and compared the verteporfin drug-light product of one and two-photon PDT.

The drug-light product for verteporfin TPE-PDT was more than three orders of magnitude higher than the corresponding value for one photon PDT. This was as expected since verteporfin has a much lower absorption cross-section for two-photon excitation. Consequently, a much higher light and drug dose would be necessary to achieve the same vascular occlusion. Interestingly, the corresponding verteporfin TPE-PDT drug-light product in the chicken CAM was ten times higher, suggesting that the CAM vasculature is much less responsive to TPE-PDT. It must be noted that the neovasculature in the CAM is very leaky compared to the mouse vasculature tested. It is quite possible that the higher drug-light product in the CAM is a consequence of lower effective drug concentrations in the excitation volume due to the leaky nature of the vessels.

The porphyrin dimer (dimer 1, fig.3) tested by Khurana et al (Khurana et al., 2009) had a 340-fold higher two-photon absorption cross-section compared to verteporfin, but exhibited only a twenty times lower drug-light product. The lower than expected effectiveness of the photosensitizer is probably due to poorer uptake and/or different localization to PDTsensitive sites in the vasculature.

\section{TPE-PDT: Challenges}

For the transition of TPE-PDT from the lab to the clinic, there are still several roadblocks to be met. The main challenges in the development of TPE-PDT are the need for: TPE-specific drugs, inexpensive lasers, adaptive optics to correct for optical aberration in the lens/cornea, and integration of technology into a "point and shoot" package for physicians.

A good TPE-specific drug needs to have high two-photon absorption, low human toxicity, high efficiency for singlet oxygen generation and should be easily targeted to the retinal neovasculature. The previously mentioned Porphyrin Dimer 1 (section 2 and fig. 3) meets at least three of these criteria. It has high two-photon absorption, very high singlet oxygen yield and shows promise for targeting to the neovasculature (Collins et al., 2008). It is, as such, a promising candidate for testing as a TPE-PDT drug.

Currently, TPE-PDT can only be achieved by excitation with expensive, high energy, pulsed lasers that can provide the high energy densities required for two-photon absorption. However, photosensitizers with very high two-photon absorption can potentially be activated by inexpensive low energy lasers, making TPE-PDT an economically viable treatment. Porphyrin dimers, with their large two-photon absorption cross-section and high efficiency of singlet oxygen generation, hold out the promise of inexpensive TPE-PDT treatment (Drobizhev et al., 2005; Kobuke \& Ogawa, 2008). 
The next challenge would be to develop adaptive optics for localized delivery of laser light into the retinal neovasculature. The laser beam needs to be tightly focused upon the macular neovasculature, and the defocusing effect of the eye-lens as well as the cornea needs to be overcome to achieve this. Research is currently under way at the Campbell laboratory, University of Waterloo, to develop an ophthalmoscope for TPE-PDT.

\section{TPE-PDT and anti-VEGF therapy}

Photodynamic therapy has proved extremely useful in prohibiting choroidal neovascular leakage and conservation of visual acuity (Arnold et al., 2001; Azab et al., 2004). It does not, however, prevent reoccurrence of retinal leakage, and treatment needs to be repeated at regular intervals (Schmidt-Erfurth et al., 1999). For effective arrest and/or cure of wet-AMD, PDT needs to be complemented by other treatments that prevent the formation of neovasculature. Of these, anti-VEGF therapy shows the greatest promise (Abouammoh \& Sharma, 2011; Chiang \& Regillo, 2011; Ozkiris, 2010). It targets VEGF-A, the vascular endothelial growth factor (VEGF) that is associated with promoting neovascularisation and angiogenesis. The role of VEGF-A in the pathogenesis of the wet-AMD is well recognized (Ferrara et al., 2003; Kliffen et al., 1997). PDT has also been implicated in the upregulation of VEGF, thereby promoting vascularisation, even as it eradicates the existing neovasculature. A treatment strategy that targets VEGF at the same time as it occludes existing neovasculature is therefore, strongly indicated.

Currently, two anti-VEGF strategies are most commonly used in treatment, both of which involve antibodies to VEGF-A (Abouammoh \& Sharma, 2011). Bevacizumab (Avastin, Genentech, San Fransisco, California, USA) is a humanized monoclonal antibody to VEGFA, while Ranibizumab (Lucentis, Genentech, San Fransisco, California, USA) is a monoclonal antibody fragment derived from Bevacizumab. Both Avastin and Lucentis are administered through intraocular injections and have been shown to significantly improve the visual acuity. In both cases, the treatment requires repeated intraocular injections and neither of them can eradicate pre-existing neovasculature. Combining anti-VEGF therapy with TPE-PDT for treating wet-AMD has the potential to reduce the number of anti-VEGF injections, while still greatly improving the visual acuity by blocking neovascularisation of the retinal macula and destroying pre-existing leakage.

Preliminary investigations of combined Avastin and one-photon PDT treatments suggest that while the combination therapy does not always improve visual acuity, it does reduce the number of retreatments required (Abouammoh \& Sharma, 2011; Chiang \& Regillo, 2011). Clinical trials are currently under way in North America and Europe to investigate the effects of combined PDT and Lucentis anti-VEGF therapy (Abouammoh \& Sharma, 2011; Chiang \& Regillo, 2011).

\section{Conclusions}

Laboratory experiments show that two-photon excitation photodynamic therapy offers potential for better selectivity and lower collateral damage in treating wet age-related macular degeneration. Experiments in the chicken embryo and mouse window models show that it is possible to achieve long-term, complete occlusion of blood vessels using twophoton excitation of verteporfin. Design of photosensitizers with higher two-photon 
absorption cross sections is likely to improve the efficacy of the TPE-PDT treatment modality in wet-AMD. Pre-clinical and clinical studies on the safety and efficacy of twophoton excitation photodynamic therapy of wet-AMD are needed before two-photon excitation photodynamic therapy is applied for clinical use.

\section{References}

Abouammoh, M. \& Sharma, S., (2011) Ranibizumab versus bevacizumab for the treatment of neovascular age-related macular degeneration. Current Opinion in Ophthalmology, 22, 152-158.

Arnold, J., Kilmartin, D., Olson, J., Neville, S., Robinson, K., Laird, A., Richmond, C., Farrow, A., McKay, S., McKechnie, R., Evans, G., Aaberg, T. M., Brower, J., Waldron, R., Loupe, D., Gillman, J., Myles, B., Saperstein, D. A., Schachat, A. P., Bressler, N. M., Bressler, S. B., Nesbitt, P., Porter, T., Hawse, P., Harnett, M., Eager, A., Belt, J., Cain, D., Emmert, D., George, T., Herring, M., McDonald, J., Mones, J., Corcostegui, B., Gilbert, M., Duran, N., Sisquella, M., Nolla, A., Margalef, A., Miller, J. W., Gragoudas, E. S., Lane, A. M., Emmanuel, N., Holbrook, A., Evans, C., Lord, U. S., Walsh, D. K., Callahan, C. D., DuBois, J. L., Moy, J., Kenney, A. G., Milde, I., Platz, E. S., Lewis, H., Kaiser, P. K., Holody, L. J., Lesak, E., Lichterman, S., Siegel, H., Fattori, A., Ambrose, G., Fecko, T., Ross, D., Burke, S., Conway, J., Singerman, L., Zegarra, H., Novak, M., Bartel, M., Tilocco-DuBois, K., Ilc, M., Schura, S., Joyce, S., Tanner, V., Rowe, P., Smith-Brewer, S., Greanoff, G., Daley, G., DuBois, J., Lehnhardt, D., Kukula, D., Fish, G. E., Jost, B. F., Anand, R., Callanan, D., Arceneaux, S., Arnwine, J., Ellenich, P., King, J., Aguado, H., Rollins, R., Anderson, T., Nork, C., Duignan, K., Boleman, B., Jurklies, B., Pauleikhoff, D., Hintzmann, A., Fischer, M., Sowa, C., et al., (2001) Verteporfin therapy of subfoveal choroidal neovascularization in age-related macular degeneration: Twoyear results of a randomized clinical trial including lesions with occult with no classic choroidal neovascularization-verteporfin in photodynamic therapy report 2. American Journal of Ophthalmology, 131, 541-560.

Azab, M., Benchaboune, M., Blinder, K. J., Bressler, N. M., Bressler, S. B., Gragoudas, E. S., Fish, G. E., Hao, Y., Haynes, L., Lim, J. I., Menchini, U., Miller, J. W., Mones, J., Potter, M. J., Reaves, A., Rosenfeld, P. J., Strong, A., Su, X. Y., Slakter, J. S., SchmidtErfurth, U. \& Sorenson, J. A., (2004) Verteporfin therapy of subfoveal choroidal neovascularization in age-related macular degeneration: Meta-analysis of 2-year safety results in three randomized clinical trials: Treatment of age-related macular degeneration with photodynamic therapy and verteporfin in photodynamic therapy study report no. 4. Retina, 24, 1-12.

Bressler, N. M. \& Bressler, S. B., (2000) Photodynamic therapy with verteporfin (visudyne): Impact on ophthalmology and visual sciences. Investigative Ophthalmology and Visual Science, 41, 624-628.

Chiang, A. \& Regillo, C. D., (2011) Preferred therapies for neovascular age-related macular degeneration. Current Opinion in Ophthalmology, 22, 199-204.

Clancy, A. A., Gregoriou, Y., Yaehne, K. \& Cramb, D. T., (2010) Measuring properties of nanoparticles in embryonic blood vessels: Towards a physicochemical basis for nanotoxicity. Chemical Physics Letters, 488, 99-111. 
Collins, H. A., Khurana, M., Moriyama, E. H., Mariampillai, A., Dahlstedt, E., Balaz, M., Kuimova, M. K., Drobizhev, M., Yang, V. X. D., Phillips, D., Rebane, A., Wilson, B. C. \& Anderson, H. L., (2008) Blood-vessel closure using photosensitizers engineered for two-photon excitation. Nature Photonics, 2, 420-424.

Drobizhev, M., Stepanenko, Y., Dzenis, Y., Karotki, A., Rebane, A., Taylor, P. N. \& Anderson, H. L., (2005) Extremely strong near-IR two-photon absorption in conjugated porphyrin dimers: Quantitative description with three-essential-states model. Journal of Physical Chemistry B, 109, 7223-7236.

Ferrara, N., Gerber, H. P. \& LeCouter, J., (2003) The biology of vegf and its receptors. Nature Medicine, 9, 669-676.

Goyan, R., Paul, R. \& Cramb, D. T., (2001) Photodynamics of latex nanospheres examined using two-photon fluorescence correlation spectroscopy. Journal of Physical Chemistry B, 105, 2322-2330.

Goyan, R. L. \& Cramb, D. T., (2000) Near-infrared two-photon excitation of protoporphyrin ix: Photodynamics and photoproduct generation. Photochemistry and Photobiology, $72,821-827$.

Gregoriou, Y. (2011) Quantum dot bioaccumulation in angiogenic tissue: Towards a physicochemical basis for nanotoxicity. PhD thesis submitted to the Department of Chemistry, University of Calgary, Calgary, Canada.

Hamblin, M. R. \& Hasan, T., (2004) Photodynamic therapy: A new antimicrobial approach to infectious disease? Photochemical and Photobiological Sciences, 3, 436-450.

Khurana, M., Collins, H. A., Karotki, A., Anderson, H. L., Cramb, D. T. \& Wilson, B. C., (2007) Quantitative in vitro demonstration of two-photon photodynamic therapy using photofrin and visudyne. Photochemical and Photobiological Sciences, 83, 14411448.

Khurana, M., Moriyama, E. H., Mariampillai, A., Samkoe, K., Cramb, D. \& Wilson, B. C., (2009) Drug and light dose responses to focal photodynamic therapy of single blood vessels in vivo. Journal of Biomedical Optics, 14, 064006.

Kliffen, M., Sharma, H. S., Mooy, C. M., Kerkvliet, S. \& de Jong, P. T., (1997) Increased expression of angiogenic growth factors in age-related maculopathy. British Journal of Ophthalmology, 81, 154-162.

Kobuke, Y. \& Ogawa, K., (2008) Recent advances in two-photon photodynamic therapy. Anti-Cancer Agents in Medicinal Chemistry, 8, 269-279.

Krammer, B., (2001) Vascular effects of photodynamic therapy. Anticancer Research, 21, 42714277.

Lou, P. J., Jones, L. \& Hopper, C., (2003) Clinical outcomes of photodynamic therapy for head-and-neck cancer. Technology in Cancer Research and Treatment, 2, 311-317.

Oheim, M., Michael, D. J., Geisbauer, M., Madsen, D. \& Chow, R. H., (2006) Principles of two-photon excitation fluorescence microscopy and other nonlinear imaging approaches. Advanced Drug Delivery Reviews, 58, 788-808.

Ozkiris, A., (2010) Anti-VEGF agents for age-related macular degeneration. Expert Opinion on Therapeutic Patents, 20, 103-118.

Patten, B. M. (1971) Early embryology of the chick. McGraw-Hill Book Company, New York.

Pervaiz, S., (2001) Reactive oxygen-dependent production of novel photochemotherapeutic agents. Faseb Journal, 15, 612-617. 
Rattner, A. \& Nathans, J., (2006) Macular degeneration: Recent advances and therapeutic opportunities. Nature Reviews Neuroscience, 7, 860-872.

Reinke, M. H., Canakis, C., Husain, D., Michaud, N., Flotte, T. J., Gragoudas, E. S. \& Miller, J. W., (1999) Verteporfin photodynamic therapy retreatment of normal retina and choroid in the cynomolgus monkey. Ophthalmology, 106, 1915-1923.

Samkoe, K. S., Fecica, M. S., Goyan, R. L., Buchholz, J. L., Campbell, C., Kelly, N. M. \& Cramb, D. T., (2006) Photobleaching kinetics of optically trapped multilamellar vesicles containing verteporfin using two-photon excitation. Photochemistry and Photobiology, 82, 152-157.

Samkoe, K. S., Clancy, A. A., Karotki, A., Wilson, B. C. \& Cramb, D. T., (2007) Complete blood vessel occlusion in the chick chorioallantoic membrane using two-photon excitation photodynamic therapy: Implications for treatment of wet age-related macular degeneration. Journal of Biomedical Optics, 12, 034025.

Samkoe, K. S. (2007) Two-photon excitation photodynamic therapy: Progress towards a new treatment for wet age-related macular degeneration. PhD thesis submitted to the Department of Chemistry, University of Calgary, Calgary, Canada.

Schlatter, P., Konig, M. F., Karlsson, L. M. \& Burri, P. H., (1997) Quantitative study of intussusceptive capillary growth in the chorioallantoic membrane (cam) of the chicken embryo. Microvascular Research, 54, 65-73.

Schmidt-Erfurth, U., Miller, J. W., Sickenberg, M., Laqua, H., Barbazetto, I., Gragoudas, E. S., Zografos, L., Piguet, B., Pournaras, C. J., Donati, G., Lane, A. M., Birngruber, R., van den Berg, H., Strong, H. A., Manjuris, U., Gray, T., Fsadni, M. \& Bressler, N. M., (1999) Photodynamic therapy with verteporfin for choroidal neovascularization caused by age-related macular degeneration: Results of retreatments in a phase 1 and 2 study. Archives of Ophthalmology, 117, 1177-1187.

Schmidt-Erfurth, U. \& Hasan, T., (2000) Mechanisms of action of photodynamic therapy with verteporfin for the treatment of age-related macular degeneration. Survey of Ophthalmology, 45, 195-214.

Skovsen, E., Snyder, J. W., Lambert, J. D. \& Ogilby, P. R., (2005) Lifetime and diffusion of singlet oxygen in a cell. Journal of Physical Chemistry B, 109, 8570-8573.

So, P. T. C., Dong, C. Y., Masters, B. R. \& Berland, K. M., (2000) Two-photon excitation fluorescence microscopy. Annual Review of Biomedical Engineering, 2, 399-429.

Soubrane, G. \& Bressler, N. M., (2001) Treatment of subfoveal choroidal neovascularisation in age related macular degeneration: Focus on clinical application of verteporfin photodynamic therapy. British Journal of Ophthalmology, 85, 483-495. 


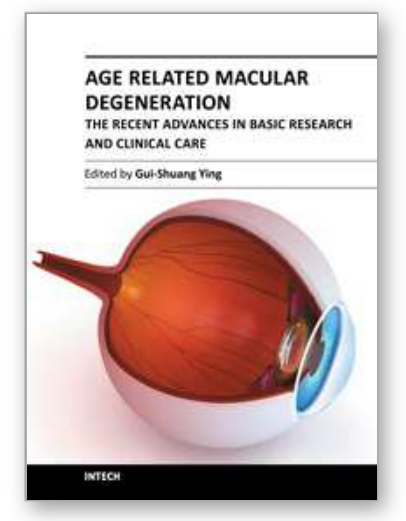

\author{
Age Related Macular Degeneration - The Recent Advances in Basic \\ Research and Clinical Care \\ Edited by Dr. Gui-Shuang Ying
}

ISBN 978-953-307-864-9

Hard cover, 300 pages

Publisher InTech

Published online 20, January, 2012

Published in print edition January, 2012

Age-related Macular Degeneration (AMD) is the leading cause of vision loss and blindness in the developed countries. In the past decade, great progress has been made in understanding the pathobiology and genetics of this blinding disease, as well as in finding new therapies for its treatment. These include the discovery of several genes that are associated with the risk of AMD, new anti-VEGF treatments for wet AMD and new imaging techniques to diagnose and monitor the AMD. All chapters in this book were contributed by outstanding research scientists and clinicians in the area of AMD. I hope this timely book will provide the basic scientists and clinicians with an opportunity to learn about the recent advances in the field of AMD.

\title{
How to reference
}

In order to correctly reference this scholarly work, feel free to copy and paste the following:

Ira Probodh and David Thomas Cramb (2012). Two-Photon Excitation Photodynamic Therapy: Working Toward a New Treatment for Wet Age-Related Macular Degeneration, Age Related Macular Degeneration The Recent Advances in Basic Research and Clinical Care, Dr. Gui-Shuang Ying (Ed.), ISBN: 978-953-307864-9, InTech, Available from: http://www.intechopen.com/books/age-related-macular-degeneration-therecent-advances-in-basic-research-and-clinical-care/two-photon-excitation-photodynamic-therapy-workingtoward-a-new-treatment-for-wet-age-related-macula

\section{INTECH}

open science | open minds

\author{
InTech Europe \\ University Campus STeP Ri \\ Slavka Krautzeka 83/A \\ 51000 Rijeka, Croatia \\ Phone: +385 (51) 770447 \\ Fax: +385 (51) 686166 \\ www.intechopen.com
}

\author{
InTech China \\ Unit 405, Office Block, Hotel Equatorial Shanghai \\ No.65, Yan An Road (West), Shanghai, 200040, China \\ 中国上海市延安西路65号上海国际贵都大饭店办公楼405单元 \\ Phone: +86-21-62489820 \\ Fax: +86-21-62489821
}


(C) 2012 The Author(s). Licensee IntechOpen. This is an open access article distributed under the terms of the Creative Commons Attribution 3.0 License, which permits unrestricted use, distribution, and reproduction in any medium, provided the original work is properly cited. 